\title{
Efecto de las podas sobre Arceuthobium spp. en bosques densos y semidensos de Pinus hartwegii (Lindl.)
}

\section{Effect of pruning on Arceuthobium spp. in dense and semi-dense forests of Pinus hartwegii (Lindl.)}

\author{
Alma I. Sotero-Garcíal, Tizbe T. Arteaga-Reyes ${ }^{*}$, Ángel R. Martínez-Campos y Leopoldo Galicia²
}

Universidad Autónoma del Estado de México. Instituto de Ciencias Agropecuarias y Rurales. El Cerrillo Piedras Blancas, México.
Universidad Nacional Autónoma de México. * Instituto de Geografía. Ciudad de México, México.
Autor de correspondencia. tizbe@hotmail.com

\section{RESUMEN}

Los muérdagos son una de las principales plagas en bosques templados de México, y las podas se han implementado como una estrategia para su control y evitar su propagación. El objetivo fue determinar el efecto de las podas sobre las poblaciones de muérdago enano (Arceuthobium spp.) en bosque semidenso y denso del área natural protegida Nevado de Toluca, México. Se seleccionaron 26 parcelas en bosques densos y semidensos con poda y sin poda. En cada parcela se determinó el diámetro normal y la altura de los árboles, así como su porcentaje y grado de infestación, comparando sitios con poda y sin poda durante tres años de evaluación. Los bosques semidenso y denso corresponden a una sucesión secundaria y dirigida, respectivamente; con diferencias estadísticas $(\mathrm{p}<0.05)$ entre la talla de los árboles podados y no podados. El porcentaje de árboles infestados por muérdago enano no mostró diferencias estadísticas significativas entre los tres periodos de evaluación, tanto en el bosque denso (4.7\%) como semidenso (12.1\%). Ambos tipos de bosque sin tratamiento fueron diferentes significativamente en el porcentaje de árboles infestados entre los tres periodos de evaluación, registrando los mayores porcentajes de árboles infestados en los grados de infección 1, 2 y 6 , siendo las clases diamétricas $\leq 20$ cm más susceptibles al ataque de este patógeno. Las podas reducen la incidencia de muérdago enano tanto en bosque semidenso como denso y su efecto fue permanente en el periodo evaluado.

PALABRAS Clave: grado de infección, muérdago enano, plagas, saneamiento forestal.

\section{ABSTRACT}

Mistletoes are one of the principal pests in temperate forests of Mexico, and pruning has been implemented as a strategy for their control and to prevent their propagation. The objective was to determine the effect of pruning on population of dwarf mistletoe (Arceuthobium spp.) in semi dense and dense forest in the natural protected area of Nevado de Toluca, Mexico. Twenty six plots were selected in dense and semi dense forests with and without pruning. In each plot, normal diameter (ND) and height $(\mathrm{H})$ of trees were determined, as well as their percentage and degree of infestation, comparing sites with and without pruning during three years of evaluation. Semi dense and dense forests correspond to a secondary and guided succession respectively, with statistical differences $(\mathrm{p}<0.05)$ between size of trees with and without pruning. Percentage of infected trees by dwarf mistletoe did not show significant statistical differences among the three evaluation periods, in dense (4.7\%) and semi dense (12.2\%) forest. Both types of forest without treatment were significantly different in the percentage of infested trees in the three periods of evaluation, registering the major percentage of infected trees in the degree of infection 1, 2 and 6 , the diameter classes $\leq 20 \mathrm{~cm}$ being more susceptible to the attack of this pathogen. Pruning reduces the incidence of dwarf mistletoe in semi dense and dense forest, and its effect was permanent during the evaluated period. 


\section{INTRODUCCIÓN}

Los muérdagos son considerados los agentes biológicos de mayor destrucción entre los bosques templados de coníferas (Geils, Cibrián y Moody, 2002). El género Arceuthobium (muérdago enano) parasita coníferas de interés comercial, causando pérdidas económicas en la producción maderable en diversas regiones en Canadá y Norte América (Hawksworth y Wiens, 1996; Reid y Shamoun, 2009; DeBruyn, Paetkau, Ross, Godfrey y Friedman, 2015). Durante el parasitismo el sistema endofítico crece y se desarrolla internamente en las ramas del hospedero, una vez que la infección se establece (dos a cinco años) emergerán los primeros brotes jóvenes donde la infección secundaria se intensifica rápidamente (Hawksworth y Wiens, 1996; Worrall y Geils, 2006). La infección reduce las tasas de crecimiento, fecundidad y transpiración; limitando la capacidad fotosintética del hospedero (Press y Phoenix, 2005; Mathiasen, Nickrent, Shaw y Watson, 2008).

El proceso de infestación se conoce como ascendente en el dosel (Hawksworth y Wiens, 1996), por lo que la densidad forestal juega un papel importante; en bosques densos la producción de brotes es suprimida por la falta de luz (Muir, Robinson y Geils, 2004). En contraste, la baja densidad arbórea estimula el desarrollo de los brotes aéreos debido a una menor competencia por los nutrientes y agua disponibles en el suelo, así como por la energía luminosa (Bickford, Kolb y Geils, 2005). El desarrollo del muérdago enano puede verse comprometido por acciones antropogénicas (manejo forestal y perturbación), alteraciones naturales (factores ambientales e incendios forestales) y características propias del bosque (edad y talla de los árboles), limitando la captación de recursos obtenidos del hospedero (Watson, 2001).

Entre las investigaciones sobre el manejo de muérdago enano se encuentran la aplicación de controles químicos que causan abscisión de brotes y la muerte foliar en un periodo de 15 días a 45 días (Coria, Vázquez, Muñoz y Villa, 2010) o el control biológico con hongos fitopatógenos que atacan la corteza y el sistema endofítico, logrando reducir la producción de brotes y semillas (Askew, Shamoun y van der Kamp, 2011; Martin, Friedman y Phillips, 2012). Por otra parte, la implementación de prácticas silvícolas como los incendios prescritos (Concklin y Geils, 2008), los clareos (Trummer, Hennon, Hansen y Muir,
1998) y la combinación de quemas y clareo (Hessburg, Povak y Brion, 2008) han logrado reducir hasta $20 \%$ de árboles infestados por muérdago enano. Sin embargo, los incendios pueden afectar el vigor de los árboles siendo más susceptibles al ataque de otras plagas y enfermedades (Hawksworth y Wiens, 1996). Se ha observado que las podas han reducido el porcentaje de árboles infestados por muérdago en abetos (Maffei et al., 2016); o bien disminuir la propagación e intensificación del muérdago enano (Shaw y Agne, 2017).

En México los muérdagos representan la segunda causa de daño forestal después de los incendios (Hawksworth, Wiens y Geils, 2002). De acuerdo con la Secretaría de Medio Ambiente y Recursos Naturales (Semarnat) en el periodo 1990-2011 el promedio anual de la superficie afectada por plagas y enfermedades forestales en el país fue de 38640 ha, de las cuales 13910 ha $(36 \%)$ corresponden a los muérdagos. Las acciones realizadas por el Programa de Conservación y Restauración de Ecosistemas Forestales (Procoref) coordinado por la Comisión Nacional Forestal (Conafor), incluyen el diagnóstico fitosanitario y posterior tratamiento a través de las labores de sanidad forestal (Semarnat, 2013).

En el Estado de México la Protectora de Bosques del Estado de México (Probosque), a través del programa de detección y combate de plagas realizó saneamientos en 651.5 ha durante el periodo 2000-2003 (Collado y Serrato, 2009). Dichos saneamientos también se han llevado a cabo en Áreas Naturales Protegidas como es el Nevado de Toluca, donde se tiene registro de la presencia de las especies Arceuthobium vaginatum (Willd.) Presl. y A. globosum (Hawksw. y Wiens) en los bosques de Pinus spp. (Franco, Endara, Regil y Nava, 2009). Durante el periodo 2009-2013 Probosque realizó la poda de ramas verdes para el control de muérdago enano en 175 ha de bosques de Pinus hartwegii (Lindl.) (Probosque, 2014); más de 70\% de la superficie tratada se ubican en la ladera norte del área natural protegida. Sin embargo, la eficiencia de los tratamientos no ha sido evaluada.

\section{OBJETIVOS}

El objetivo fue determinar el efecto de las podas sobre las poblaciones de Arcenthobium spp. del área natural protegida Nevado de Toluca, México. 


\section{MATERIALES Y MÉTODOS}

\section{Área de estudio}

El estudio se llevó a cabo dentro del área natural protegida Nevado de Toluca (NT) localizada en los 1851’31" y $19^{\circ} 19^{\prime} 03^{\prime \prime}$ latitud Norte y 99³8'54” y 10009'58” longitud Oeste (Rojas, Valdez, Mireles, Reyes y Pastor, 2007), en los ejidos San José Contadero y Santa María del Monte (Fig. 1) ubicados en la ladera Norte (altitud promedio de $3700 \mathrm{~m}$ ) dentro del municipio de Zinacantepec. Los bosques de pino (Pinus spp.) predominan arriba de los $2900 \mathrm{~m}$ de altitud, el clima es semifrío subhúmedo C (E) wig (2800 m - $3700 \mathrm{~m} \mathrm{snm}$ ) con una temperatura media anual entre $-2{ }^{\circ} \mathrm{C} y+7{ }^{\circ} \mathrm{C}$, lluvias en verano y una precipitación media anual de $1000 \mathrm{~mm}$ (González et al., 2007).

\section{Diseño de muestreo}

Se procedió a un muestreo estratificado dirigido considerando como estratos los rodales tratados y no tratados, la muestra para cada estrato fue proporcional en cada tipo de bosque considerado, por lo tanto, en bosque semidenso se seleccionaron seis parcelas con poda y cinco sin poda, mientras que en bosque denso la superficie tratada fue mayor, por lo que se seleccionaron nueve parcelas con poda y siete sin poda. El tamaño de la muestra se obtuvo siguiendo las fórmulas propuestas por Hernández, Fernández-Collado y Baptista (2006); las parcelas fueron circulares de $1000 \mathrm{~m}^{2}$ (Flores, Rodríguez, Meléndez y Rosas, 2008), separadas entre sí $100 \mathrm{~m}$ y ubicadas en sitios con baja perturbación.

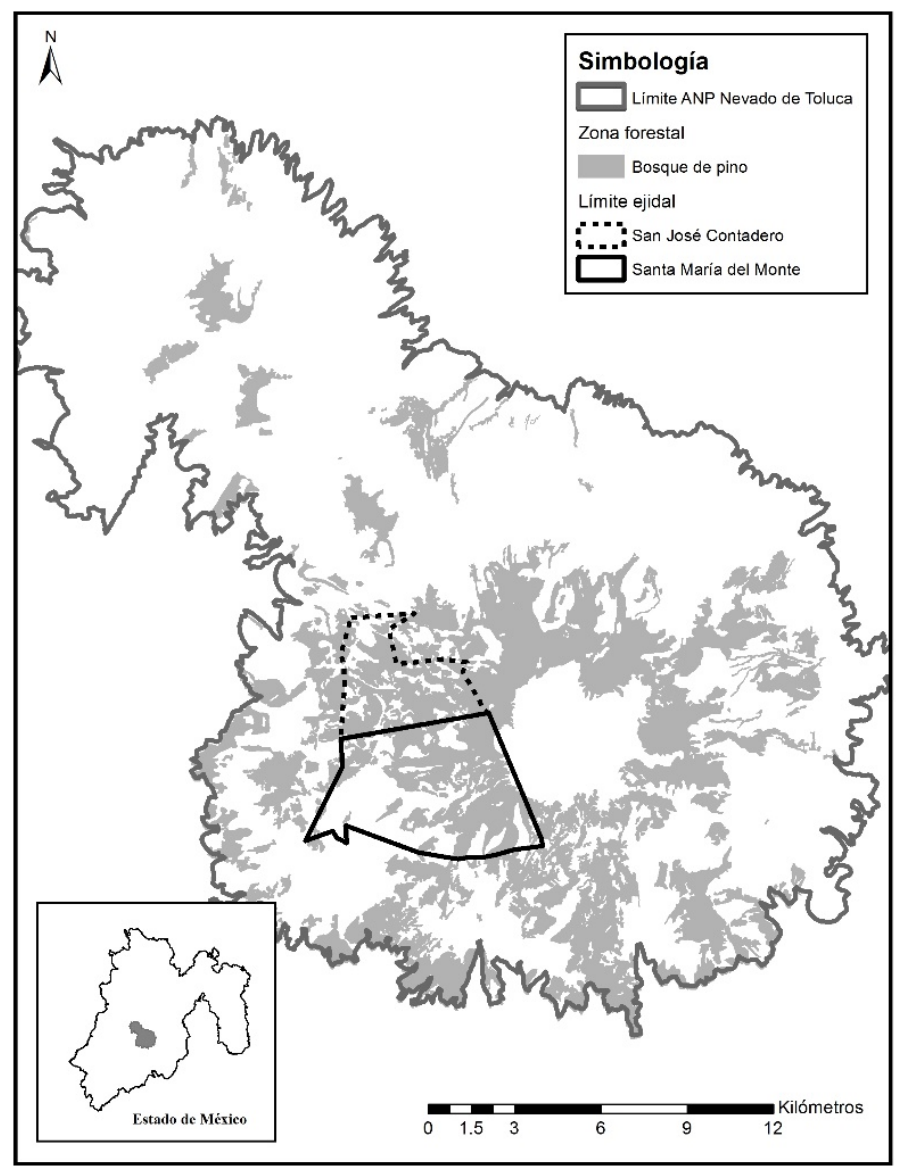

FIGURA 1. Ubicación de los ejidos de estudio dentro del ANP Nevado de Toluca 


\section{Monitoreo de variables}

Las evaluaciones se realizaron durante los meses de mayo a junio de 2013, 2014 y 2015. En cada una de las parcelas se registró el diámetro normal $(\mathrm{DN}) \geq 2.5 \mathrm{~cm}$ de todos los árboles con una altura $(A) \geq 1.5 \mathrm{~m}$; los individuos menores se registraron y solo se diferenciaron las plántulas $(\mathrm{A}<0.3 \mathrm{~m})$. Esta información se utilizó para determinar la densidad forestal, así como la distribución horizontal. Asimismo, se registró la presencia y ausencia de muérdago enano para determinar el porcentaje de árboles infestados y su distribución por grado de infección. La severidad de la infección se determinó de acuerdo con el método propuesto por Hawksworth (1977). Este sistema se basa en la estimación visual de la intensidad de la infección en la copa, la cual se divide en tres secciones verticales (alta, media y baja); para cada una se determina la intensidad: 0 = infección no visible, $1=$ $<50 \%$ de ramas infectadas y $2=>50 \%$ de ramas infectadas. La intensidad de infección para cada tercio se suma para determinar el grado de infección para el árbol: $0=$ nulo, $1-2=$ leve, 3-4 = moderado y 5-6 = severo.

\section{Análisis estadístico}

Para las variables DN, A, porcentaje de árboles infestados y grado de infección, se efectuó la prueba de normalidad y homogeneidad de varianza Kolmogorov Smirnov. Debido a la distribución de los datos se eligieron los análisis no paramétricos de Friedman para $\mathrm{k}$ muestras relacionadas, para determinar las diferencias entre los años de evaluación por tratamiento y entre tratamientos para ambos tipos de bosque $(\mathrm{p}<0.05)$. Los análisis fueron realizados mediante el paquete estadístico SPSS ${ }^{\circledR}$ v. 20.0. (IBM, 2011) para Windows 7.

\section{RESULTADOS Y DISCUSIÓN}

\section{Características de los bosques}

La población del bosque semidenso fue de 233 árboles/ha; la distribución de los valores de DN de los árboles muestra que las plántulas y los individuos de categoría $<5$ comprenden 54\% de la población (Fig. 2a). Galeana-Pizaña, Ordoñez-Díaz y CoronaRomero (2013) sugiere que este tipo de distribución diamétrica corresponde a un proceso de sucesión dirigida de individuos en etapas de regeneración avanzada, resultado de las campañas de reforestación como se ha observado en bosques de Abies religiosa (Kunth Schltdl. Et Cham.) y Pinus hartwegii (Lindl.). En el bosque denso la población arbórea fue de 486 árboles/ha, la distribución de diámetros indica que la media de los individuos se encuentra en la clase diamétrica de $5 \mathrm{~cm}$ (Fig. 2b), sugiriendo una sucesión secundaria correspondiente a un estado de regeneración activa de individuos del género Quercus spp y P. pseudostrobus Lindl. que han sobrevivido a incendios (Alanís-Rodríguez et al., 2011).
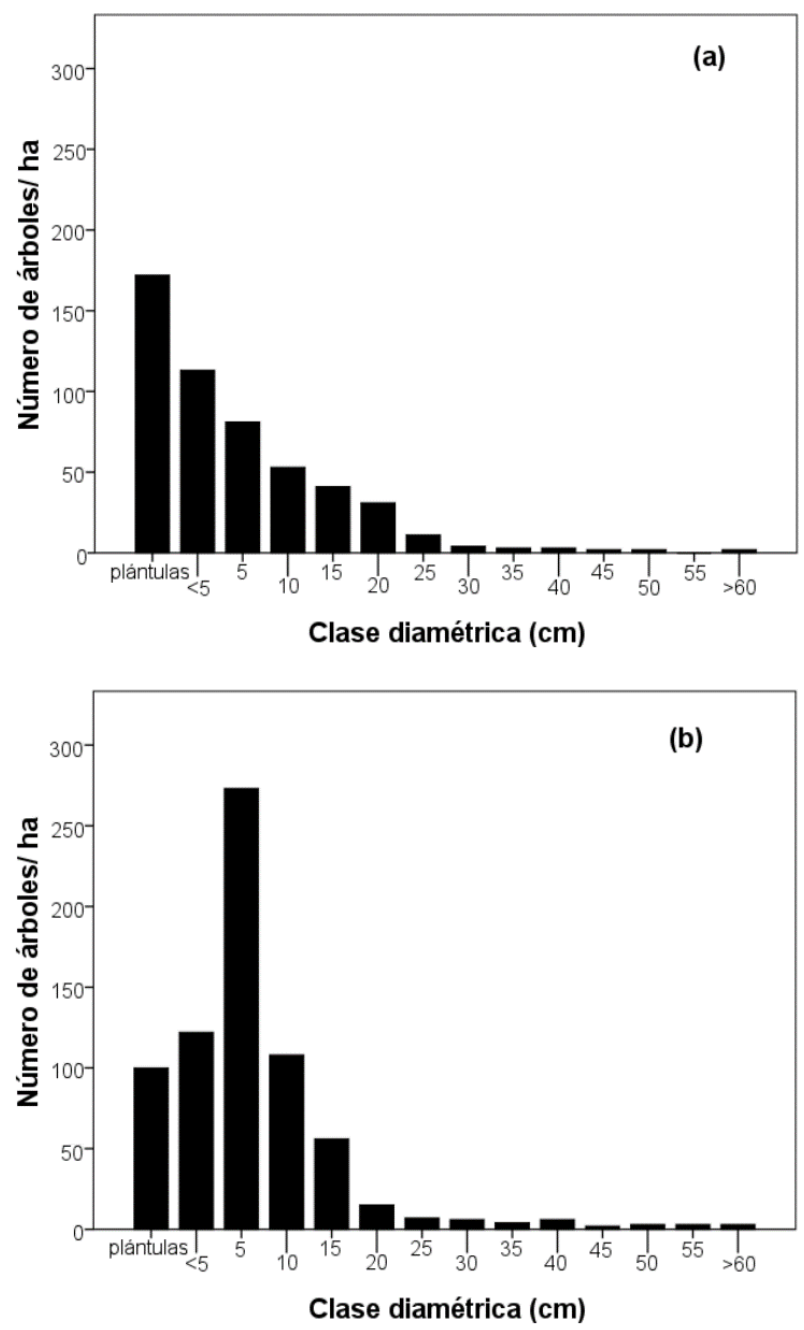

Figura 2. Distribución horizontal de Pinus hartwegii (Lindl.) en bosque semidenso (a) y denso (b).

En bosques semidensos la media del diámetro normal y altura de la población de pinos podados fue $80 \%$ y $56 \%$, respectivamente, mayor que el valor correspondiente a los 
individuos sin poda; mientras que en los bosques densos las podas solo promovieron $40 \%$ de incremento en el diámetro normal y $27 \%$ de incremento en altura (Tabla 1). Las diferencias estadísticas $(p<0.05)$ en la talla muestra que la reducción en la biomasa de la copa por efecto de la poda reactiva la capacidad fotosintética (Amateis y Burkhart, 2011), reduce la competencia de requerimientos nutrimentales entre la copa y el tallo, lo que favorece el crecimiento y desarrollo de los árboles.

\section{Grado de infección en árboles no tratados}

Los bosques no tratados presentaron diferencias estadísticas $(\mathrm{p}<$ 0.05) en los tres periodos de evaluación. Para el bosque semidenso el porcentaje de árboles infestados incrementó 16.1\% (Tabla 2), debido a que la baja densidad arbórea estimula el desarrollo de los brotes aéreos y por ende la propagación del muérdago enano (Bickford et al., 2005). En contraste, el bosque denso registró una disminución en el porcentaje de árboles infestados (Tabla 3), debido a la inclusión de individuos en la categoría $<5(32.2 \%)$ que no fueron considerados en la primera evaluación, ya que no cumplían con la talla (DN) y altura considerada para el presente estudio, sin embargo, en las subsecuentes evaluaciones se incorporaron.

El porcentaje de árboles infestados por muérdago enano es consistente con lo hallado en otros estudios realizados en bosques de Pinus hartwegii (Lindl.) del centro del país parasitados por $A$. globosum Hawksw. y Wiens y A. vaginatum (Willd.) Presl. (Hernández-Benítez, Cano-Santana, Castellanos-Vargas, 2005; Ramírez-Dávila y Porcayo-Camargo, 2010; Queijeiro-Bolaños y Cano-Santana, 2015). Por ejemplo, Ramírez-Dávila y PorcayoCamargo (2010) señalaron como valor máximo 94.8\% de árboles infestados por muérdago enano y como valor mínimo 69.6\% en diez sitios de monitoreo en bosques de Pinus motezumae Lamb. en el Parque Nacional Nevado de Toluca, sin diferenciar sitios con o sin poda.

TABLA 1. Talla promedio de los árboles por tipo de bosque.

\begin{tabular}{llllll}
\hline \multirow{2}{*}{ Tipo de bosque } & Tratamiento & \multicolumn{2}{l}{ Diámetro normal $(\mathrm{cm})$} & \multicolumn{2}{l}{ altura $(\mathrm{m})$} \\
& & Media & $E e^{*}$ & Media & $E e^{*}$ \\
\hline Semidenso & Poda & 22.7 & 1.08 & 10.5 & 0.54 \\
& Sin poda & 12.0 & 0.48 & 6.7 & 0.26 \\
Denso & Poda & 11.6 & 0.41 & 5.6 & 0.20 \\
& Sin poda & 8.4 & 0.32 & 4.4 & 0.15 \\
\hline
\end{tabular}

*Ee = error estándar.

TABLA 2. Distribución del grado de infección en bosque semidenso.

\begin{tabular}{|c|c|c|c|c|c|c|c|}
\hline \multirow{2}{*}{$\begin{array}{l}\text { Evaluaciones por } \\
\text { tratamiento }\end{array}$} & \multicolumn{7}{|c|}{ Porcentaje de árboles por grado de infección ${ }^{*}$} \\
\hline & 0 & 1 & 2 & 3 & 4 & 5 & 6 \\
\hline \multicolumn{8}{|l|}{ Poda } \\
\hline 1 & 81.9 & 4.2 & 4.2 & 1.4 & 6.9 & 0.0 & 1.4 \\
\hline 2 & 86.0 & 3.5 & 4.7 & 1.2 & 1.2 & 1.2 & 2.3 \\
\hline 3 & 95.7 & 4.3 & 0.0 & 0.0 & 0.0 & 0.0 & 0.0 \\
\hline \multicolumn{8}{|l|}{ Sin Poda } \\
\hline 1 & 43.7 & 23.0 & 21.8 & 4.6 & 0.0 & 0.0 & 6.9 \\
\hline 2 & 37.3 & 13.3 & 15.7 & 12.0 & 6.0 & 2.4 & 13.3 \\
\hline 3 & 27.6 & 13.8 & 13.8 & 3.4 & 17.2 & 0.0 & 24.1 \\
\hline
\end{tabular}


TABLA 3. Distribución del grado de infección en bosque denso.

\begin{tabular}{lccccccc}
\hline $\begin{array}{l}\text { Evaluaciones por } \\
\text { tratamiento }\end{array}$ & \multicolumn{7}{l}{ Porcentaje de árboles por grado de infección } \\
& 0 & 1 & 2 & 3 & 4 & 5 & 6 \\
\hline Poda & & & & & & & \\
\hline 1 & 86.7 & 5.7 & 3.0 & 1.8 & 0.6 & 0.3 & 1.8 \\
2 & 96.0 & 0.9 & 0.9 & 0.9 & 0.0 & 0.0 & 0.9 \\
3 & 96.0 & 0.0 & 0.0 & 0.0 & 0.0 & 0.0 & 4.0 \\
\hline Sin Poda & & & & & & & \\
\hline 1 & 28.4 & 12.7 & 10.6 & 7.5 & 2.8 & 1.3 & 36.7 \\
2 & 43.8 & 13.5 & 20.2 & 10.1 & 4.5 & 0.0 & 7.9 \\
3 & 42.4 & 11.8 & 18.8 & 10.6 & 2.4 & 2.4 & 11.8 \\
\hline
\end{tabular}

*O = sano; 1 y 2 = leve; 3 y 4 = moderada; 5 y 6 = severa.

Los mayores porcentajes de árboles infestados en el bosque semidenso se presentan en los grados de infección 1, 2 (moderado) y 6 (severo) (Tablas 2 y 3), y este último representa un riesgo de infección latente debido a que se incrementa con la densidad, ya que se favorece la dispersión de las semillas a través del viento (Hawksworth y Wiens, 1996; Muir y Geils, 2002). Los menores porcentajes de árboles infestados tanto en bosques semidensos como en bosques densos se concentran en las categorías 3, 4 y 5, y corresponde a las fracciones I (alta) y II (media) de la copa del árbol. Lo anterior, se debe probablemente a que la radiación solar puede acelerar el desarrollo del muérdago y, por ende, su propagación, por lo que se ha sugerido que la densidad arbórea limita el desarrollo del muérdago y su propagación, ya sea por limitar la incorporación de luz o debido a la competencia por nutrientes dada por el mayor número de individuos. Lo anterior es consistente con el hecho de que el muérdago enano es una planta que necesita luz para su desarrollo. Muir, Robinson y Geils (2004) mencionan que en bosques densos el muérdago se distribuye hacia la parte alta de la copa, sin embargo, la producción de brotes en estos sitios es suprimido por la falta de luz y sus efectos en el hospedero son limitados.

Tanto en bosques semidensos como densos las clases diamétricas $\leq 20 \mathrm{~cm}$ fueron las más susceptibles a ser infestados por muérdago enano, ya que se presentaron todos los grados de infección (Fig. 3a y 3b). Esto refleja que las defensas en las coníferas dependen del estado ontogénico (Lorio, Stephen y Paine, 2004) y la fenología de la planta, siendo en las etapas juveniles la máxima competencia por los recursos para su desarrollo. Por ejemplo, Pinus pinaster Ait. es atacado por insectos durante las primeras etapas de vida (Chen, Kolb y Clancy, 2002); mientras que en etapas maduras los caracteres defensivos tienen gran trascendencia para garantizar su contribución en la siguiente generación (Sampedro, Moreira y Zas, 2011).

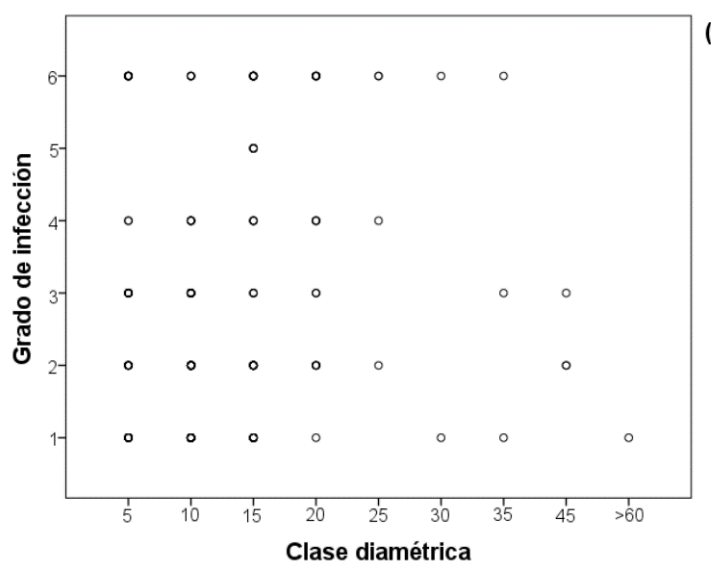

(a)

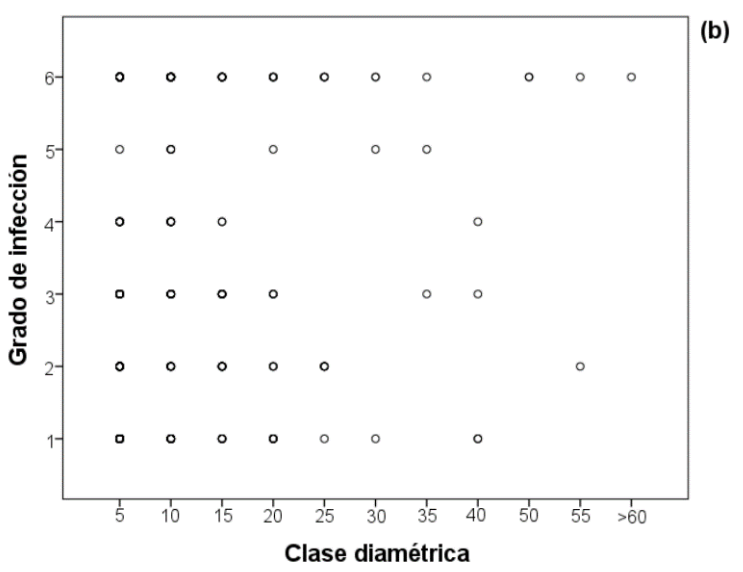

FIGURA 3. Distribución del grado de infección en bosque semidenso (a) y denso (b). 


\section{CONCLUSIONES}

Las podas son una estrategia que reduce la incidencia de muérdago enano y tiene un efecto permanente en el periodo evaluado tanto en el bosque semidenso como en el denso. Los árboles infestados de clase $\leq 20$ son más susceptibles al ataque de muérdago enano y no tienen correlación con los grados de infección. La densidad arbórea limita el desarrollo del muérdago y su propagación porque limita la incorporación de luz y la competencia por nutrientes dada por el mayor número de individuos. Las categorías juveniles son más susceptibles a la infección que las categorías adultas debido al desarrollo de mecanismos de defensa especializados.

\section{RECONOCIMIENTOS}

La autora principal agradece al Consejo Nacional de Ciencia y Tecnología (Conacyt) por el financiamiento para los estudios de posgrado en el Doctorado en Ciencias Agropecuarias y Recursos Naturales. A la Protectora de Bosques del Estado de México (Probosque) y a los Comisariados Ejidales de San José Contadero y Santa María del Monte, Estado de México, por el apoyo brindado para el desarrollo de esta investigación.

\section{REFERENCIAS}

Alanís-Rodríguez, E., Jiménez-Pérez, J., Valdecantos-Dema, A., PandoMoreno, M., Aguirre-Calderón, O., \& Treviño-Garza, E. J. (2011). Caracterización de regeneración leñosa post-incendio de un ecosistema templado del parque ecológico Chipinque, México. Revista Chapingo serie ciencias forestales y del ambiente, 17(1), 31-39. doi: 10.5154/r.rchscfa.2010.05.032

Amateis, R. L., \& Burkhart, H. E. (2011). Growth of young loblolly pine trees following pruning. Forest Ecology and Management, 262, 23382343. doi: 10.1016/j.foreco.2011.08.029

Askew, S. E., Shamoun, S. F., \& van der Kamp, B. J. (2011). Assessment of Colletotrichum gloeosporioides as a biological control agent for management of hemlock dwarf mistletoe (Arceuthobium tsugense). Forest Pathology, 41(6), 444-452. doi: 10.1111/j.14390329.2010 .00698

Bickford, C. P., Kolb, T. E., \& Geils, B. W. (2005). Host physiological condition regulates parasitic plant performance: Arceuthobium vaginatum subsp. cryptopodum on Pinus ponderosa. Oecologia, 146(2), 179-189. doi:10.1007/s00442-005-0215-0

Chen, Z., Kolb, T. E., \& Clancy, K. M. (2002). The role of monoterpenes in resistance of Douglas fir to western spruce budworm defoliation. Journal of Chemical Ecology, 28(6), 897-920. doi: 10.1023/A:1015297315104

Collado, E., \& Serrato, P. J. (2009). El sector forestal en la actualidad. En GEM [Gobierno del Estado de México] (ed.), La diversidad biológica del Estado de México. Estudio de Estado (p. 331-334). Toluca: GEM.

Concklin, D. A., \& Geils, B.W. (2008). Survival and sanitation of dwarf mistletoe-infected ponderosa pine following prescribed underburning. Western Journal of Applied Forestry, 23(4), 216-222. doi:

https://www.fs.fed.us/rm/pubs_other/rmrs_2008_conklin_d0 01.pdf

Coria, V. M., Vázquez, I., Muñoz, H. J., \& Villa, J. (2010). Impacto de tierra de diatomeas sobre Arcenthobium globosum Hawksworth \& Wiens subsp. grandicaule en Pinus pseudostrobus Lind. Revista Mexicana de Ciencias Forestales, 1(1), 39-46.

DeBruyn, R. A., Paetkau, M., Ross, K. A., Godfrey, D. V., \& Friedman, C. R. (2015). Thermogenesis-triggered seed dispersal in dwarf mistletoe. Nature Communications, 6, 6262. doi: $0.1038 /$ ncomms 7262

Flores, J. G, Rodríguez, O. G., Meléndez, M., \& Rosas, O. G. (2008). Sitios de muestreo para el monitoreo de combustibles forestales SIMMCOF. Jalisco, México: Instituto Nacional de Investigaciones Forestales, Agrícolas y Pecuarias.

Franco, S., Endara, A., Regil, H., \& Nava, G. (2009). Estudio fitosanitario forestal del Parque Nacional Nevado de Toluca. Reporte Técnico. Toluca, México: Universidad Autónoma del Estado de México.

Galeana-Pizaña, J. M., Ordóñez-Díaz, J. A. B., \& Corona-Romero, N. (2013). Estimación de contenido de carbono en la cuenca del río Magdalena, México. Madera y Bosques, 19(1), 53-69. doi: 10.21829/myb.2013.191347

Geils, B. W, Cibrián, J. T., \& Moody, B. (2002). Mistletoes of North American conifers. General Technical Report 98. Ogden, Estados Unidos: U.S. Department of Agriculture, Forest Service, Rocky Mountain Research Station.

González, A., González, A., Hernández, S. L., Madrigal, D., Morales, C., \& Pineda, N. (2007). Atlas del Medio Físico del Estado de México. Toluca, México: Cuarta Época 50.

Hawksworth, F. G., \& Wiens, D. (1996). Dwarf Mistletoes: Biology, Pathology, and Systematics. Agricultural Handbook 709. Washington D.C., Estados Unidos: U.S. Dept. of Agriculture, Forest Service.

Hawksworth, F. G. (1977). The 6-class dwarf mistletoe rating system. General Technical Report RM-48. Fort Collins, Estados Unidos: USDA Forest Service, Rocky Mountain Forest and Range Experiment Station. Recuperado de https://archive.org/details/CAT1083736

Hawksworth, F. G., Wiens, D. y Geils, B. W. (2002). Arceuthobium in North America. En Geils, B. W, Cibrían, J. y Moody, B. (Eds.), 
Mistletoes of North American conifers. General Technical Report 98 (pp. 29-56). Ogden, Estados Unidos: U.S. Department of Agriculture, Forest Service, Rocky Mountain Research Station,

Hernández, R., Fernández-Collado, C. y Baptista, P. (2006). Metodología de la Investigación (4a ed.). Ciudad de México, México: McGrawHill.

Hernández-Benítez, R., Cano-Santana, Z. y Castellanos-Vargas, I. (2005). Incidencia de infestación de Arceuthobium globosum grandicaule (Hawks. y Wiens) en Pinus hartwegii (Lindl.). Ciencia Forestal en México, 30(97), 79-86.

Hessburg, P. F., Povak, N. A. y Brion, S. R. (2008). Thinning and prescribed fire effects on dwarf mistletoe severity in an Eastern Cascade Range dry forest, Washington. Forest Ecology and Management, 255(7), 2907-2915. doi: 10.1016/j.foreco.2008.01.066

International Business Machines Corp Released [IBM] (2011). IBM SPSS Statistics for Windows, Version 20.0. Armonk, Estados Unidos: IBM Corp.

Lorio, P. L., Stephen, F. M. y Paine, T. D. (2004). Environment and ontogeny modify loblolly pine response to induced acute water deficits and bark beetle attack. Forest Ecology and Management, 73, 97-110. doi: https://doi.org/10.1016/0378-1127(94)03500-V

Maffei, H. M., Filip, G. M., Grulke, N. E., Oblinger, B. W., Margolis, E., \& Chadwick, K. L. (2016). Pruning high-value Douglas-fir can reduce dwarf mistletoe severity and increase longevity in Central Oregon. Forest Ecology and Management, 379, 11-19. doi: 10.1016/j.foreco.2016.07.014

Martin, L. L., Friedman, C., \& Phillips, L. A. (2012). Fungal endophytes of the obligate parasitic dwarf mistletoe Arceuthobium americanum (Salantaceae) act antagonistically in vitro against the native fungal pathogen Cladosporium (Davidiellaceae) of their host. American Journal of Botany, 99(12), 2017-2034. doi: 10.3732/ajb.1200189

Mathiasen, R. L., Nickrent, D. L., Shaw, D. C., \& Watson, D. M. (2008). Mistletoes: pathology, systematics, ecology, and management. Plant Disease, 92(7), 988-1006. doi: 10.1094/PDIS-92-7-0988

Muir, J. A., \& Geils, B. W. (2002). Management strategies for dwarf mistletoe: silviculture. En Geils, B. W., Cibrían, J., \& Moody, B. (Eds.), Mistletoes of North American conifers. General Technical Report 98 (pp. 83-94). Ogden, Estados Unidos: U.S. Department of Agriculture, Forest Service, Rocky Mountain Research Station.

Muir, J. A., Robinson, D. C., \& Geils, B. W. (2004). Characterizing the effects of dwarf mistletoe and other diseases for sustainable forest management. Journal of Ecosystems and Management, 3(2), 88-94. doi: http://www.forrex.org/jem/2004/vol3/no2/art6.pdf

Press, M. C., \& Phoenix, G. K. (2005). Impacts of parasitic plants on natural communities. New Phytologist, 166(3), 737-751. doi:10.1111/j.1469-8137.2005.01358.x
Protectora de Bosques del Estado de México [Probosque]. (2014). Resultados del combate y control de muérdago. Metepec, México: Probosque.

Queijeiro-Bolaños, M. E., \& Cano-Santana, Z. (2015). Dinámica temporal de la infestación por muérdago enano (Arceuthobium globosum y $A$. vaginatum) en Zoquiapan (Parque Nacional Iztaccíhuatl Popocatépetl), México. Ciencia UAT, 9(2), 06-14.

Ramírez-Dávila, J. F., \& Porcayo-Camargo, E. (2010). Estudio comparativo de la distribución espacial del muérdago enano (Arcenthobium sp.) en la ladera norte del Parque Nacional Nevado de Toluca, México. Bosque (V aldivia), 31(1), 28-38.

Reid, N., \& Shamoun, S. F. (2009). Contrasting research approaches to managing mistletoes in commercial forests and wooded pastures. Botany, 87(1), 1-9. doi:10.1139/B08-109

Rojas, E. E., Valdez, M. E., Mireles, P., Reyes, A., \& Pastor, J. (2007). Estimación de la producción de agua del Parque Nacional Nevado de Toluca para el año 2006. Quivera, 9(1), 159-176.

Sampedro, L., Moreira, X., \& Zas, R. (2011). Costs of constitutive and jasmonate-induced pine tree chemical defenses emerge only under low nutrient availability. Journal of Ecology, 99, 818-827. doi: 10.1111/j.1365-2745.2011.01814.x

Secretaría del Medio Ambiente y Recursos Naturales [Semarnat]. (2013). Informe de la situación del medio ambiente en México. Compendio de estadísticas ambientales. Indicadores clave y de desempeño ambiental. Edición 2012. Recuperado de http://apps1.semarnat.gob.mx/dgeia/informe_12/pdf/Informe _2012.pdf

Shaw, D. C., \& Agne, M. C. (2017). Fire and dwarf mistletoe (Viscaceae: Arceuthobium species) in western North America: contrasting Arceuthobium tsugense and Arceuthobium americanum. Botany, 95(3), 231-246. doi: dx.doi.org/10.1139/cjb-2016-0245

Trummer, L. M., Hennon, P. E., Hansen, E. M., \& Muir, P. S. (1998). Modeling the incidence and severity of hemlock dwarf mistletoe in 110-year-old wind-disturbed forests in Southeast Alaska. Canadian Journal of Forest Research, 28(10), 1501-1508. doi: $10.1139 / \mathrm{x} 98-132$

Watson, D. M. (2001). Mistletoe-A keystone resource in forests and woodlands worldwide. Annual Review of Ecology and Systematics, 32, 219-49. doi: 10.1146/annurev.ecolsys.32.081501.114024

Worrall, J., \& Geils, B. (2006). Dwarf mistletoes: The Plant Health Instructor. Recuperado de www.apsnet.org/edcenter/intropp/lessons/miscellaneous/Page s/Dwarfmistletoes.aspx

Manuscrito recibido el: 7 de junio de 2017

Aceptado el: 1 de diciembre de 2017

Publicado el: 15 de junio de 2018 
Este documento se debe citar como:

Sotero-García, A. I., Arteaga-Reyes, T. T., Martínez-Campos, A. R., \& Galicia, L. (2018). Efecto de las podas sobre Arceuthobium spp. en bosques densos y semidensos de Pinus hartwegii (Lindl.). Madera y Bosques, 24(2), e2421582. doi: 10.21829/myb.2018.2421582
Madera y Bosques por Instituto de Ecología, A.C. se distribuye bajo una Licencia Creative Commons Atribución-NoComercialCompartirlgual 4.0 Internacional. 